Supporting Information

\title{
Influence of microstructure topography on the oblique impact dynamics of drops on superhydrophobic surfaces
}

Damon G. K. Aboud, Anne-Marie Kietzig

Department of Chemical Engineering, McGill University, Montreal QC, H3A 0C5 Canada 


\section{Supporting Note 1:}

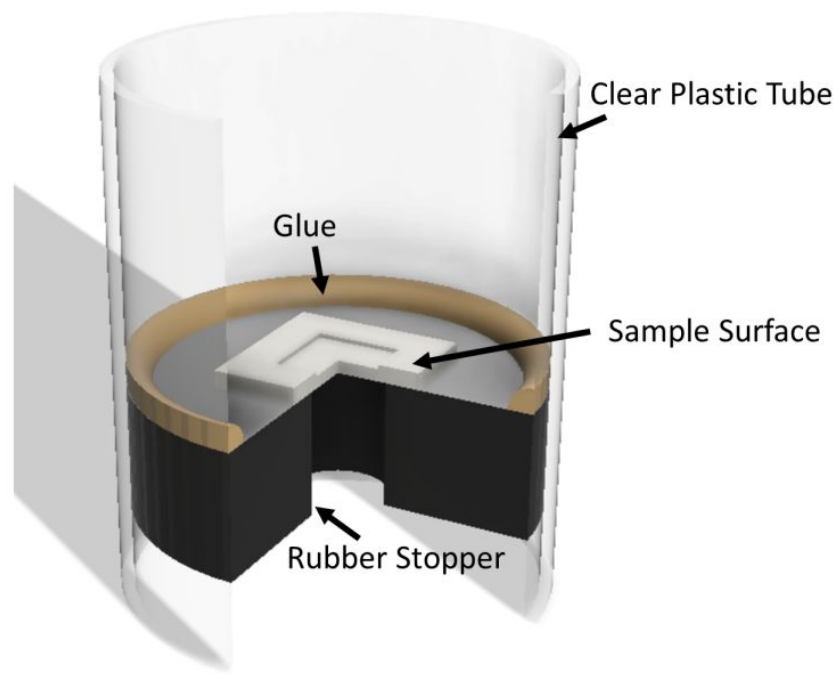

Figure S1. Schematic of the sample holder used to contain the P200 sample during immersion confocal microscopy.

Figure S1 shows a schematic of the sample holder used to obtain immersion confocal microscopy images of the P200 sample surface, which are shown in Figure 8 of the main text. A clear plastic tube is used to contain the entire assembly, so that water can be added above the sample surface to the desired depth, allowing the user to adjust the hydrostatic pressure of the experiment. In our initial attempts at this imaging technique, we had problems with air expansion and bubble formation due to the energy that the laser scanning confocal microscope delivers to the surface during imaging. To surpass this issue, we used the femtosecond laser that was used to micromachine all of our sample surfaces to drill 50 um holes through several locations on the sample. Then, we glued the sample onto a rubber stopper that had a large hole in the middle. This allowed the expanding air within the pores of the SHP surface to escape through the sample, into the cavity beneath. The rubber stopper is glued into the plastic tube, as shown in Figure S1. 


\section{Supporting Note 2:}

\begin{tabular}{|c|cccc|}
\hline \multicolumn{5}{|c|}{ Nano } \\
\hline$W e_{n} \backslash A O I$ & $0^{\circ}$ & $30^{\circ}$ & $45^{\circ}$ & $60^{\circ}$ \\
\hline 78 & Partial Reb. & Rebound & Partial Reb. & Partial Reb. \\
45 & Rebound & Rebound & Rebound & Partial Reb. \\
24 & Rebound & Rebound & Rebound & Rebound \\
10 & Rebound & Rebound & Rebound & Rebound \\
3 & Rebound & Rebound & Rebound & Rebound \\
\hline
\end{tabular}

\begin{tabular}{|c|cccc|}
\hline \multicolumn{5}{|c|}{ Wavy } \\
\hline$W e_{n} \backslash A O I$ & $0^{\circ}$ & $30^{\circ}$ & $45^{\circ}$ & $60^{\circ}$ \\
\hline 78 & Rebound & Rebound & Partial Reb. & Partial Reb. \\
45 & Rebound & Rebound & Rebound & Rebound \\
24 & Rebound & Rebound & Rebound & Rebound \\
10 & Rebound & Rebound & Rebound & Rebound \\
3 & Rebound & Rebound & Rebound & Rebound \\
\hline
\end{tabular}

\begin{tabular}{|c|cccc|}
\hline \multicolumn{5}{|c|}{ H200 } \\
\hline$W e_{n} \backslash A O I$ & $0^{\circ}$ & $30^{\circ}$ & $45^{\circ}$ & $60^{\circ}$ \\
\hline 78 & Rebound & Rebound & Rebound & Partial Reb. \\
45 & Rebound & Rebound & Rebound & Rebound \\
24 & Rebound & Rebound & Rebound & Rebound \\
10 & Rebound & Rebound & Rebound & Rebound \\
3 & Rebound & Rebound & Rebound & Rebound \\
\hline
\end{tabular}

\begin{tabular}{|c|cccc|}
\hline \multicolumn{5}{|c|}{ P100 } \\
\hline$W e_{n} \backslash A O I$ & $0^{\circ}$ & $30^{\circ}$ & $45^{\circ}$ & $60^{\circ}$ \\
\hline 78 & Rebound & Streaming & Streaming & Streaming \\
45 & Rebound & Rebound & Rebound & Partial Reb. \\
24 & Rebound & Rebound & Rebound & Rebound \\
10 & Rebound & Rebound & Rebound & Rebound \\
3 & Rebound & Rebound & Rebound & Rebound \\
\hline
\end{tabular}

\begin{tabular}{|c|cccc|}
\hline \multicolumn{5}{|c|}{ P200 } \\
\hline$W e_{n} \backslash A O I$ & $0^{\circ}$ & $30^{\circ}$ & $45^{\circ}$ & $60^{\circ}$ \\
\hline 78 & Splashing & Partial Reb. & Streaming & Streaming \\
45 & Pancake & Pancake & Pancake & Pancake \\
24 & Pancake & Pancake & Pancake & Pancake \\
10 & Rebound & Rebound & Rebound & Rebound \\
3 & Rebound & Rebound & Rebound & Rebound \\
\hline
\end{tabular}

Table S1. Map of the impact behaviour observed on all experimental surfaces, at each Weber number and angle of incidence tested. 


\section{Supporting Note 3:}
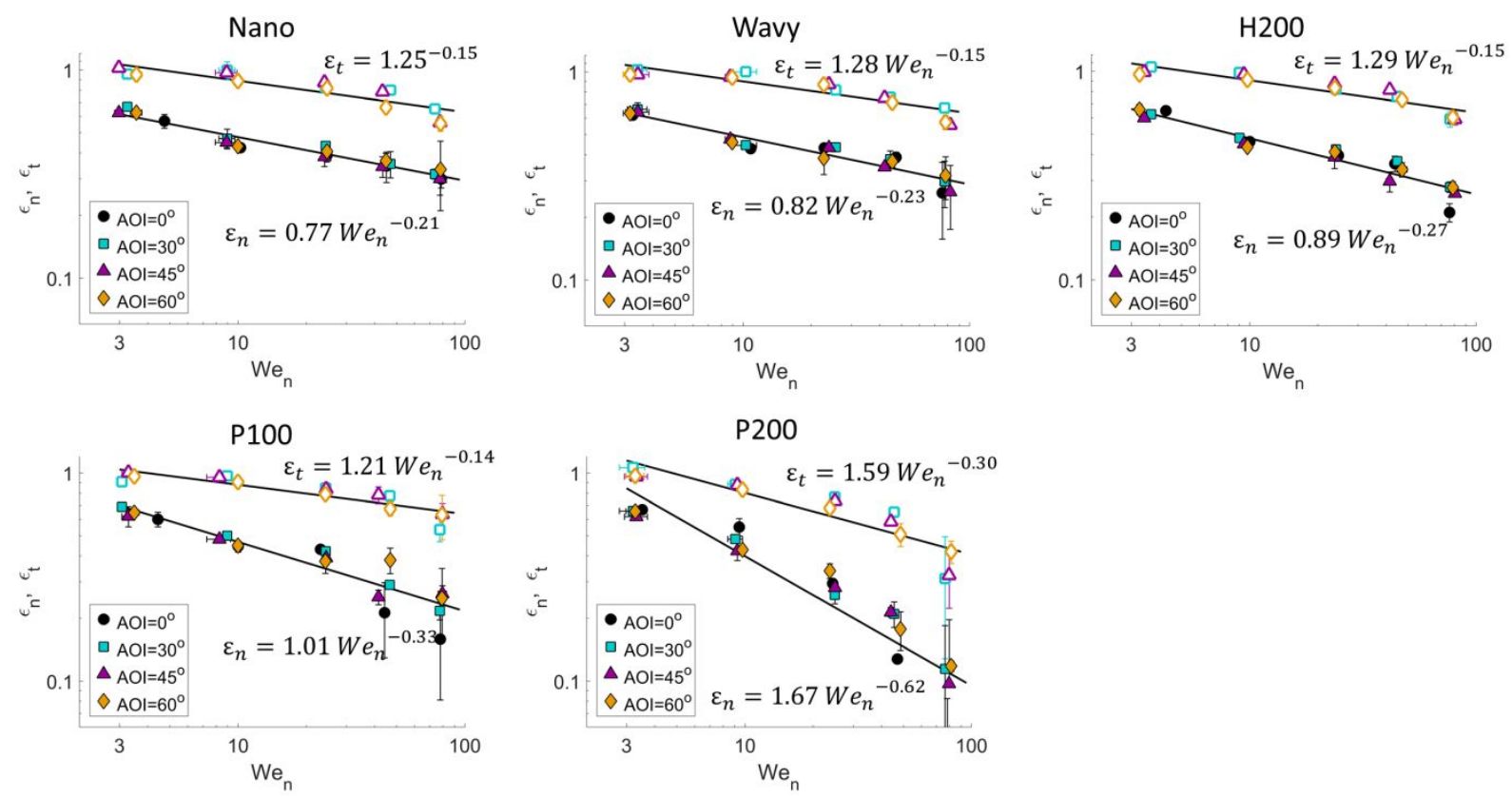

Figure S2. Normal and tangential restitution coefficients versus the normal Weber number.

Open markers indicate $\varepsilon_{t}$, and closed markers indicate $\varepsilon_{n}$. 


\section{Supporting Note 4:}

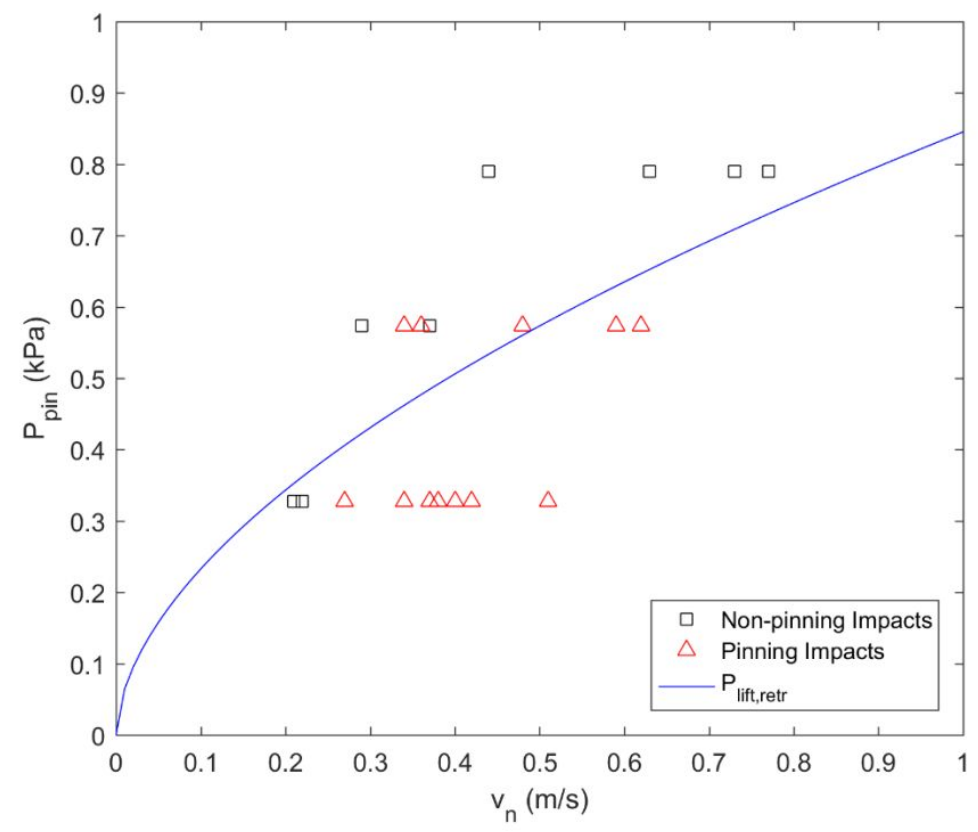

Figure S3. Critical pinning pressure versus normal drop impact speed, using data points borrowed from Lee at al. (2015).

Above, we show data points from a report by Lee at al. [1]. Lee tested drop impacts on several pillared SHP surfaces, and discovered that pinning can occur not only during the initial impact of the drop, but also during the retraction phase. In Figure S3, $P_{p i n}$ is the critical pressure that causes drop pinning based on the geometry of the sample. Lee performed a derivation for the lifting pressure during the retraction phase, and arrived at the correlation: $P_{\text {lift,retr }}=3.54 \cdot v_{n 1}$ $\sqrt{\rho \sigma / D_{0}}$. However, in Lee's derivation, they assumed that the restitution coefficient is constant as $W e_{n}$ is varied, which is not supported by our results. Therefore, following the same logic as Lee's derivation, but including $\varepsilon_{n} \propto v_{n}{ }^{-0.42}$, we arrived at a similar equation in the Restitution Coefficient section of our article: $P_{\text {lift,retr }}=k \cdot v_{n 1} \sqrt[0.58]{\rho \sigma / D_{0}}$. Then, to determine the value of $k$, we plotted Lee's results in Figure S3, and found that a value of $k=4.5$ fits well with the data. This is the value presented in the main article. 


\section{Supporting Note 5:}

In the main article, we explained that while the drop slides tangentially across the SHP surface during the expansion and retraction phases, it experiences some adhesion to the surface, caused by sites of solid-liquid contact. This adhesion acts to stretch the droplet, causing its maximum spreading diameter to increase. Based on this stretching mechanism, in a previous report we derived the following model [2]:

$$
D_{\text {max }}^{*}=D_{n, \max }^{*}+c_{0} \cdot \tan (A O I) \cdot W e_{n}{ }^{3 / 2}
$$

\section{Equation S1}

The second term of Equation S1 represents the stretching length, and accounts for the stretching mechanism described above. The variable $c_{0}$ is the adhesion coefficient and quantifies the adhesive properties of the SHP surface.

However, over the course of this study, we discovered that this model fails when the drop diameter is changed. Specifically, in our previous report, we observed the impact of $1.29 \mathrm{~mm}$ diameter water droplets impacting only the Wavy surface, and found that using an adhesion coefficient of $c_{0}=9.99 \times 10^{-4}$, Equation S1 was highly accurate in predicting $D_{\max }^{*}$ [2] However, in this report we used a larger drop diameter of $2.04 \mathrm{~mm}$, and using the same sample surface, we found that $c_{0}=5.65 \times 10^{-4}$ (both graphs are shown below in Figure S4). Hence, the adhesion coefficient, which should remain constant for a given surface (since it quantifies the adhesion properties), appears to vary with drop diameter. This problem occurs because the Weber number, which is included in Equation S1, is proportional to the drop diameter $\left(W e_{n}=\rho v_{n 1}{ }^{2} D_{0} / \sigma\right.$ ). To remedy this problem, we have performed a revised derivation of the model, with emphasis placed on the dynamic pressure of impact $\left(P_{d}=\rho v_{n 1}{ }^{2}\right)$ instead of on $W e_{n}$. This change is sensible, since previous reports have demonstrated that liquid penetration into the microstructure is actually proportional to $P_{d}$, instead of $W e_{n}$ [3]. And increased liquid penetration creates additional sites of liquid-solid contact, leading to greater adhesion.

This derivation follows the same steps as were presented in our previous article (Aboud and Kietzig, "On the Oblique Impact Dynamics of Drops on Superhydrophobic Surfaces. Part I: Sliding Length and Maximum Spreading Diameter", 2018) [2]. We assumed that the maximum spreading diameter of an oblique drop impact is equal to the sum of two quantities: the spreading diameter that would be reached by a drop impacting at normal $A O I\left(D^{*}{ }_{n, \max }\right)$, and the 
dimensionless stretching length $\left(S L^{*}\right)$ that results from the drop's adhesion to the surface as it slides tangentially down the tilted sample:

$$
D^{*}{ }_{\max }=D^{*}{ }_{n, \max }+S L^{*}
$$

Equation S2

From observation, we know that the stretching length of the drop increases for drops with a greater sliding length on the surface $\left(L_{0}{ }^{*}\right)$, and also for drops that experience more adhesion on the surface, which is related to partial penetration of the liquid into the porous structure of the SHP surface. For this reason, in the original derivation we postulated that $S L^{*}$ is proportional to the product of $L_{0}{ }^{*}$ and the normal Weber number, which could quantify the liquid penetration $\left(S L^{*}\right.$ $\left.\propto L_{0}{ }^{*} \cdot W e_{n}\right)$. However, this argument is flawed since previous reports have demonstrated that liquid penetration is actually proportional to the dynamic pressure of impact $P_{d} \propto \rho v_{n 1}{ }^{2}$, instead of the Weber number [3]. Therefore, in this revised derivation, we assert that the stretching length is in fact proportional to the product of $L_{0}{ }^{*}$, and the dynamic impact pressure $P_{d}$ :

$$
S L^{*} \propto L_{0}{ }^{*} P_{d} \propto L_{0}{ }^{*} \rho v_{n 1}{ }^{2}
$$

Equation S3

This relationship is proven graphically in Figure S4(c) using sliding length data from our 2018 publication [2].

To make Equation S3 usable, $L_{0}{ }^{*}$ must be stated as a function of known quantities. The sliding length is approximately equal to $L_{0}{ }^{*}=v_{t 1} t_{c} / D_{0}$. And, as demonstrated by Richard et al. (2002), the contact time of a rebounding drop $\left(t_{c}\right)$ is governed by the scaling relationship $t_{c} \propto$ $\sqrt{\rho D_{0}{ }^{3} / \sigma}$. Therefore, the dimensionless sliding length of an impacting drop is proportional to:

$$
L_{0}{ }^{*} \propto v_{t 1} \sqrt{\rho D_{0} / \sigma}
$$

Equation S4

Substituting $v_{t 1}=v_{n 1} \cdot \tan (A O I)$, we find that $L_{0}{ }^{*}$ can be stated as:

$$
L_{0}^{*} \propto \tan (A O I) \sqrt{W e_{n}}
$$

Equation S5

Then, substituting Equation S5 into Equation S3, the stretching length can be stated in terms of known quantities:

$$
S L^{*} \propto \tan (A O I) \sqrt{W e_{n}}\left(\rho v_{n 1}^{2}\right)
$$

Equation S6

Substituting Equation S6 into Equation S2, we arrive at the following revised expression for the dimensionless maximum spreading diameter of oblique drop impacts that is presented in the main article as Equation 8:

$$
D_{\text {max }}^{*}=D_{n, \max }^{*}+c \cdot \tan (A O I) \sqrt{W e_{n}}\left(\rho v_{n 1}{ }^{2}\right)
$$

Equation 8 
where $c$ is the adhesion coefficient.

Figure S4 demonstrates the importance of this revised derivation. Using Equation S1, the value of the adhesion coefficient $c_{0}$ changes by a factor of nearly 2 when the diameter of the drop changes from $1.29 \mathrm{~mm}$ to $2.04 \mathrm{~mm}$. However, using Equation 8, the value of the revised adhesion coefficient $c$ changes by only $9 \%$ when the diameter is changed, indicating much better robustness of the model to changes in drop impact conditions.

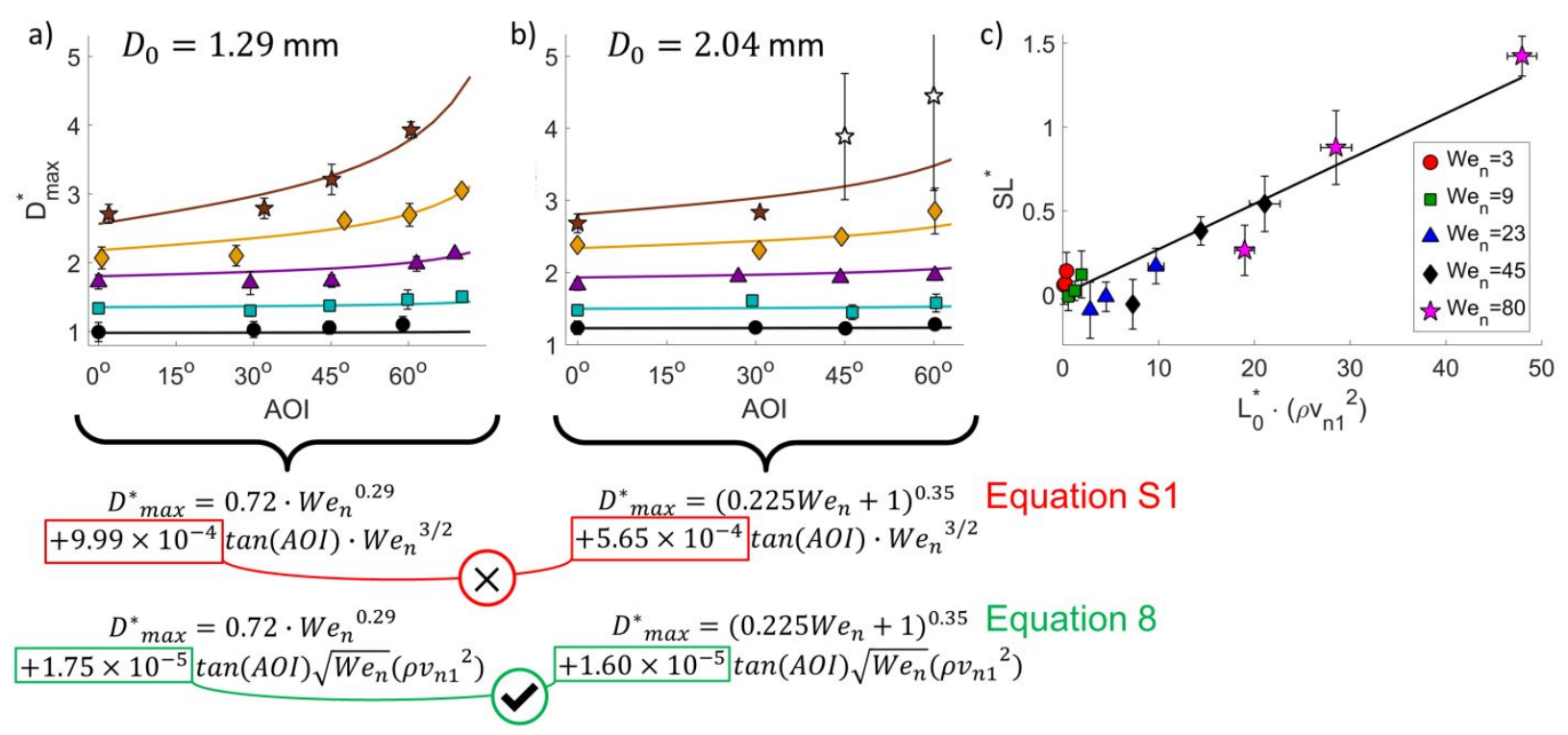

Figure S4. (a-b) Graphs of the dimensionless maximum spreading diameter of drops impacting the Wavy surface for 2 different drop diameters. Possible model fits are presented under each graph. (c) Dimensionless stretching length versus the product of the dimensionless sliding length and the dynamic impact pressure. 


\section{Supporting Note 6:}

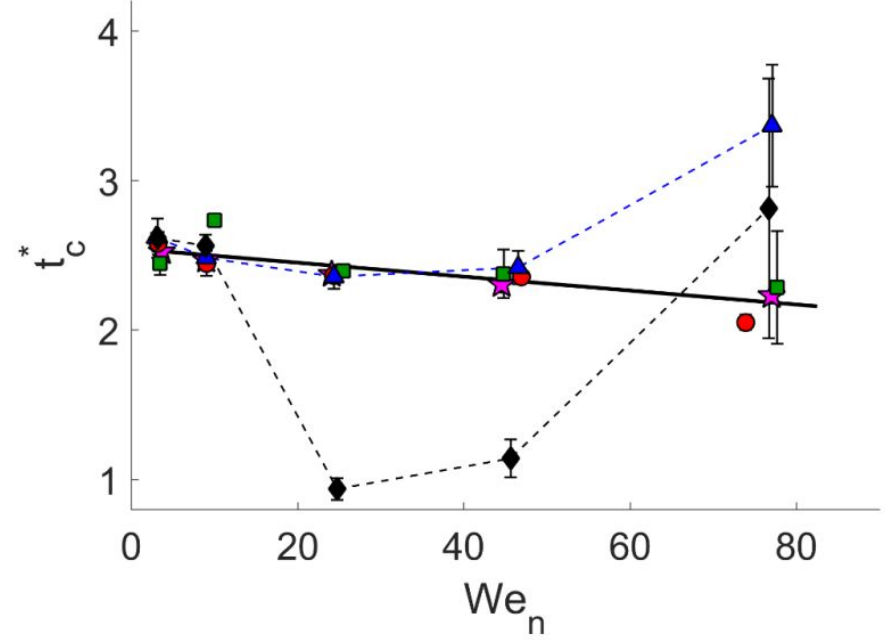

Figure S5. Evolution of the dimensionless contact time as $W e_{n}$ is raised for $A O I=30^{\circ}$. 


\section{$\underline{\text { References: }}$}

1. Lee, C.; Nam, Y.; Lastakowski, H.; Hur, J. I.; Shin, S.; Biance, A.-L.; Pirat, C.; Ybert, C. Two Types of Cassie-to-Wenzel Wetting Transitions on Superhydrophobic Surfaces During Drop Impact. Soft Matter 2015, 11, 4592-4599.

2. Aboud, D. G.; Kietzig, A.-M. On the Oblique Impact Dynamics of Drops on Superhydrophobic Surfaces. Part I: Sliding Length and Maximum Spreading Diameter. Langmuir 2018, 34, 9879-9888.

3. LeClear, S.; LeClear, J.; Park, K.-C.; Choi, W. Drop Impact on Inclined Superhydrophobic Surfaces. J. Colloid Interface Sci. 2016, 461, 114-121. 